\title{
Comparison between parameters of muscle performance and inflammatory biomarkers of non-sarcopenic and sarcopenic elderly women
}

This article was published in the following Dove Press journal:

Clinical Interventions in Aging

2 August 2017

Number of times this article has been viewed

\section{Lygia Paccini Lustosa' \\ Patrícia Parreira Batista' \\ Daniele Sirineu Pereira ${ }^{2}$ \\ Leani Souza Máximo \\ Pereira' \\ Aline Scianni \\ Giane Amorim Ribeiro- \\ Samora'}

'Physical Therapy Department, Universidade Federal de Minas Gerais, Belo Horizonte, ${ }^{2}$ Physical Therapy Department, Universidade Federal de Alfenas, Alfenas, Brazil
Correspondence: Lygia Paccini Lustosa Departamento de Fisioterapia, EEFFTO, Universidade Federal de Minas Gerais, Avenida Antônio Carlos, 6627, CEP 31270 90I, Belo Horizonte, Minas Gerais, Brazil

Tel +55 3l 34094783

Email llustosa@ufmg.br
Background: Sarcopenia is a multifactorial geriatric syndrome with complex interrelationships. Increased plasma levels of inflammatory mediators increase the catabolic stimuli of the musculature, thereby causing a decrease in mass and muscular function.

Objective: The objective of this study was to compare the performance of the knee extensors test (by isokinetic dynamometer) and plasma levels of interleukin-6 (IL-6) and soluble receptors of tumor necrosis factor alpha (sTNFR1) between sarcopenics and non-sarcopenics communitydwelling elderly women residents of Brazil.

Material and methods: The diagnosis of sarcopenia included measurements of body composition (by densitometry with dual energy source of X-ray), handgrip strength (by Jamar ${ }^{\circledR}$ dynamometer), and the usual gait velocity according to the recommendations of the European Working Group on Sarcopenia in Older People. In both sarcopenics and non-sarcopenics elderly women, we evaluated the muscle function by knee extensors test (using an isokinetic dynamometer Byodex System 4 Pro $^{\circledR}$ ) at angular speeds of $60^{\circ} / \mathrm{s}$ and $180 \%$ s) and also we evaluated the plasma concentrations of IL-6 and sTNFR1. Comparisons of muscle performance between groups were carried out using mixed factorial ANOVA with post hoc Bonferroni test; sTNFR1 and IL-6 variables were analyzed by applying Mann-Whitney $U$ test.

Results: Statistical differences were observed between groups regarding muscle power $(P=0.01)$, total work adjusted to body weight $(P=0.01)$ at a rate of $180^{\circ} / \mathrm{s}$, and plasma levels of sTNFR1 $(P=0.01)$.

Conclusion: Sarcopenic elder women showed lower performance of the lower limbs, especially at a higher speed, predisposing these older women to greater vulnerability in functional activities that require agility and postural stability. Plasma levels of sTNFR1 were higher for non-sarcopenics elderlies. However, due to the observational nature of the study, it was impossible to infer causality among the variables surveyed.

Keywords: sarcopenia, muscle performance, inflammatory mediators, aging

\section{Introduction}

Population aging is a global reality. It is known that changes in the health profile, chronic degenerative diseases, increased vulnerability to functional limitation, loss of physical independence, and quality of life are associated with the advancement of age. ${ }^{1}$ In this panorama, sarcopenia stands out, which is considered a multifactorial geriatric syndrome with complex interrelationships. In addition, sarcopenia is difficult to be diagnosed and it presents with adverse health effects throughout the clinical course, such as functional disability, falls, osteoporosis, frailty, hospitalizations, and death. ${ }^{2}$ It affects more often women, since a high proportion of women live longer than men. 
The European Working Group on Sarcopenia in Older People (EWGSOP), in 2010, published recommendations on the definition and operational criteria for the diagnosis of sarcopenia. ${ }^{3}$ The authors defined sarcopenia as progressive and generalized loss of muscle mass and muscle function associated with aging, and they proposed an algorithm for identifying sarcopenia with specific cutoffs for each parameter. Therefore, a sarcopenic elderly, as defined by the EWGSOP, presents with decreased mobility and/or loss of muscle strength and reduced muscle mass. ${ }^{3}$ According to this algorithm, the prevalence of sarcopenia among the Brazilian elderlies reached $17 \%,{ }^{4}$ of whom 1\%-29\% were community elderlies and 14\%-33\% institutionalized elderlies. ${ }^{5}$ This broad variation in prevalence is due to the absence of a consensus on its definition and diagnostic criteria, choice of instruments, and indices adopted in the respective studies. ${ }^{4,5}$

Associated changes in aging predispose the development of sarcopenia, such as aging, loss of quantity and quality of muscle fibers (especially type II fibers), decreased response of motor neurons, mitochondrial dysfunction, lipid infiltration and connective tissues, hormonal and metabolic changes, and changes in immunological responses. ${ }^{3,6}$ In particular, the immunological changes indicate a high concentration of inflammatory mediators in plasma levels. ${ }^{7}$

Increased plasma levels of circulating inflammatory mediators increase the catabolic stimuli of the musculature, thereby reflecting a decrease in muscle mass and function. ${ }^{7,8}$ Tumor necrosis factor (TNF-alpha) is a pro-inflammatory cytokine, with short length, that acts directly and indirectly on muscle degradation process, induces the production of other catabolic cytokines such as interleukin-6 (IL-6), and triggers a second inflammatory cycle. ${ }^{8,9}$ Studies have reported that the receptor - soluble receptors of tumor necrosis factor alpha (sTNFR1) - acts as an initial inflammatory marker, with higher stability in plasma levels of IL-6 and sTNFR that reflects on the TNF-alpha behavior. ${ }^{9,10}$ Another mediator often investigated in epidemiological studies and related to aging is IL-6. It is involved in both pro- and anti-inflammatory processes and is associated with multiple adverse health conditions and functional disability. ${ }^{7}$

Although several studies have reported significant association of these inflammatory mediators with muscle parameters, controversial results exist in the literature, which confirm the absence of a consensus on the catabolic behavior of mediators. ${ }^{1-13}$ In addition, most studies have withheld the outcome of sarcopenia, failing to investigate the interrelationship between muscle mass and muscle function with these biomarkers in the elderlies. However, there is a lack of information regarding the plasma levels in sarcopenic and non-sarcopenic elderlies and how they differ. Similarly, the characteristics of different parameters of muscular performance - torque peak, power, work normalized by body weight in that condition - are also unknown.

In this context, the aim of this study was to compare the performance of knee extensors (using isokinetic dynamometer at angular speeds of $60 \%$ s and $180 \%$ s) and plasma levels of IL-6 and sTNFR1 between sarcopenic and non-sarcopenic community-dwelling elderlies in Brazil.

\section{Materials and methods}

This is an observational cross-sectional study approved by the Research Ethics Committee of Universidade Federal de Minas Gerais, Brazil (CAAE 39702014.2.0000.5149). The elderlies were from a Reference Center care unit. The eligible elderlies answered a questionnaire with sociodemographic and clinical information and signed the free and informed consent. In a second moment, data collection included evaluations of blood sample, muscle performance, and body compositions.

\section{Sample}

Our sample consisted mainly of elderly women classified as non-sarcopenic elderly (NSE) and sarcopenic elderly (SE). Inclusion criteria were as follows: $\geq 65$ years, sedentary for a period of at least $\geq 3$ months, and residents of the community irrespective of race and/or class.

Exclusion criteria included cognitive impairment detected by the Mini Mental State Examination according to schooling, ${ }^{14}$ neurological and/or rheumatologic self-reported diseases or sequelae, acute pain that prevented the tests from being performed, dependent gait, history of cancer in the past 5 years, history of knee or hip arthroplasty, and use of corticoids (immunosuppressant medication).

\section{Instruments and measures}

Sarcopenia was diagnosed according to the guidelines of EWGSOP; it included loss of muscle mass and reduced muscle function, which is given by a reduction in muscular strength and/or a decline in muscular function. ${ }^{3}$ For the gait speed test, participants walked a distance of $8.6 \mathrm{~m}$, timing the middle $4.6 \mathrm{~m}$. The initial $2 \mathrm{~m}$ (considered as acceleration) and the final $2 \mathrm{~m}$ (considered deceleration) were discarded (reliability of 0.90 and 0.91 for comfortable and fast speeds, respectively). ${ }^{15,16}$ We considered the average of three test repetitions $(\mathrm{m} / \mathrm{s})$ for analysis. Elderlies with an 
average gait speed $\leq 0.8 \mathrm{~m} / \mathrm{s}$ had function limitation and were recommended for body composition measurements. Those with gait speed $>0.8 \mathrm{~m} / \mathrm{s}$ performed the handgrip strength test. If the handgrip strength was $<20$ kilogram/force ( $\mathrm{kg} / \mathrm{f})$, they had to perform body composition tests. ${ }^{3}$

The handgrip strength test was performed with Jamar ${ }^{\circledR}$ dynamometer, according to the protocol of the American Society of Hand Therapists for positioning and execution. ${ }^{16}$ The test was performed in the dominant limb, considered the one uses to sign. For the elderlies to attain maximum isometric strength, the researchers clapped their hands and used standard phrases to motivate them. Three replications of the test were recorded at an interval of 1 minute, and the average of these values was expressed in $\mathrm{kg} / \mathrm{f}$.

We used densitometry with dual energy X-ray source (dual-energy X-ray absorptiometry [DXA]; Hologic Discovery $\mathrm{W}$ model and software version 3.3.01) to measure body compositions and evaluate muscle mass. The DXA identifies body components enabling the indirect evaluation of lean mass, fat mass, and bone mass. ${ }^{18}$ The skeletal muscle mass index $\left(\mathrm{ALM} / \mathrm{h}^{2}\right)$ refers to the sum of the muscle mass of the four limbs divided by square height. ${ }^{3,17}$ The cutoff point reported by Baumgartner et al was adopted for calculating muscle loss, ie, values $<5.45 \mathrm{~kg} / \mathrm{h}^{2}$ for women. ${ }^{3,18,19}$

The muscle performance of the lower limbs was evaluated by isokinetic dynamometer Byodex System 4 Pro $^{\circledR}$ (Biodex Systems, Shirley, NY, USA) at angular speeds of $60 \%$ and $180 \%$, with 5 and 15 repetitions, respectively. Prior to the test, the elderly women received instructions and walked for 5 minutes at their usual speed as a warm-up. Then, they sat in the chair and the strips were adjusted. They performed five repetitions at an angular velocity of $60 \%$ s so as to train themselves; then they started the test with a focus to optimize their maximum strength. To motivate the elderly during the test, the researchers clapped their hands and gave standardized phrases to optimize their performance. The average torque peak data, average power, and total work of the knee extensors in a concentric contraction mode were extracted and normalized by body weight, with regard to the two speeds tested. A protocol used in previous studies by our research group was adopted to ensure the quality of information extracted..$^{20}$

Blood was always collected in the morning between 8 and 10 hours so as to avoid possible variations in circadian cycle. ${ }^{21}$ From the ulnar vein, $5 \mathrm{~mL}$ of blood was extracted in a vacuum tube with EDTA. The blood obtained was centrifuged (Fanem Ltd., São Paulo, Brazil) at $1,500 \mathrm{rpm} .{ }^{20}$ The plasma was collected in microtubes and stored in a freezer at $-80^{\circ} \mathrm{C}$. The concentrations of inflammatory mediators were analyzed by enzyme-linked immunosorbent assay (ELISA), using a high-sensitivity kit for IL-6 (Quantikine ${ }^{\circledR}$ HS ELISA; R\&D Systems, Minneapolis, MN, USA) and DuoSet kit ELISA for sTNFR1 (R\&D Systems). The readings were obtained by a microplate reader set to $490 \mathrm{~nm}$, with a wavelength correction at $650 \mathrm{~nm}$.

\section{Statistical analysis}

For the calculation of sample size, based on a previous pilot study, we considered variable gait speed, statistical power of $80 \%$, confidence interval of $95 \%$, and error of $20 \%$. As a result, 26 participants were included in each group. We added $20 \%$ due to the possibility of loss during the study; therefore, each group consisted of 32 elderlies.

Socioclinical and demographic characteristics were analyzed by measuring central tendency and dispersion (mean and standard deviation) and categorical variables were measured by absolute and relative frequencies. The Shapiro-Wilk test was performed to verify data distribution. Comparisons between the NSE and SE groups for muscle performance were carried out by mixed factorial analysis of variance (ANOVA) with post hoc Bonferroni test. sTNFR1 and IL-6 variables were measured by nonparametric Mann-Whitney $U$ test. The Statistical Package for Social Sciences (SPSS, version 17) was used to analyze $95 \%$ confidence intervals with a confidence level of $5 \%$.

\section{Results}

We screened 389 elderly women, of whom 122 met the study inclusion criteria and the algorithm proposed by EWGSOP. After the evaluation of muscle mass, from 122 elderly included in the study only 63 elderly women remained in the sample: 32 classified as non-sarcopenic, and 31 as sarcopenic (Figure 1).

Comparison of characteristics between the groups showed no statistical difference $(P>0.05)$ between the parameters analyzed, except for the variables body mass index (BMI) and number of comorbidities (Table 1). It is noteworthy that according to the algorithm adopted in this study for the diagnosis of sarcopenia (EWGSOP), only the skeletal muscle mass index $\left(\mathrm{ALM} / \mathrm{h}^{2}\right)$ and gait speed were statistically different between the groups, which shows a greater discriminatory impact of gait speed on handgrip strength in this geriatric syndrome. Regarding the associated chronic diseases, the groups self-reported the following: heart disease (NSE 27.3\% and SE 15.6\%), diabetes (NSE 27.3\% and SE $6.3 \%$ ), arthritis (NSE $42.4 \%$ and SE $18.8 \%$ ), systemic arterial hypertension (NSE 69.7\% and SE 62.5\%), respiratory disease (NSE 21.2\% and SE 0\%), cataracts (NSE 57.6\% 


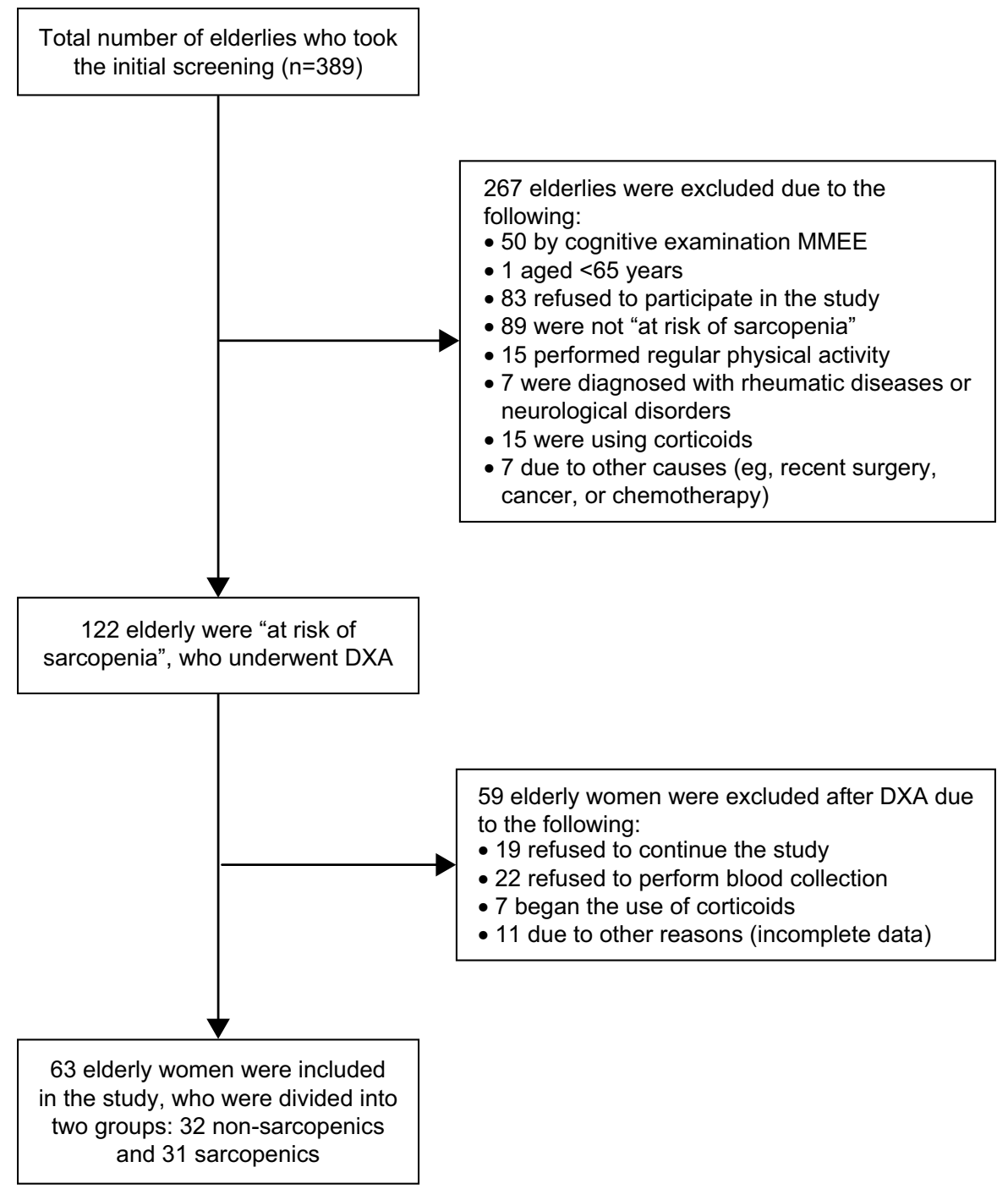

Figure I Flowchart of study participants.

Abbreviations: DXA, dual-energy X-ray absorptiometry; MMEE, Mini Mental State Examination.

Table I Characterization of the groups, demonstrating average, mean, and standard deviation of quantitative descriptive variables and the variables marital status, race, and education, the highest frequency reported

\begin{tabular}{llll}
\hline Characteristics & NSE $(\mathbf{n}=\mathbf{3 2})$ & SE $(\mathbf{n}=\mathbf{3 I})$ & $\boldsymbol{P}$-value \\
\hline Age (years) & $77.15(6.9 \mathrm{I})$ & $77.18(5.42)$ & $0.7 \mathrm{I}$ \\
Education (years) & $2.76(2.56)$ & $3.69(2.82)$ & 0.13 \\
BMI $\left(\mathrm{kg} / \mathrm{m}^{2}\right)$ & $28.48(5.3 \mathrm{I})$ & $21.55(2.28)$ & $0.0 I^{*}$ \\
Comorbidities (n) & $2.82(\mathrm{I} .59)$ & $\mathrm{I} .94(\mathrm{I} .2 \mathrm{I})$ & $0.02^{*}$ \\
Medications (n) & $4.5 \mathrm{I}(2.38)$ & $3.69(\mathrm{I} .97)$ & 0.14 \\
Caloric expenditure & $\mathrm{I}, 247.03(2,483.25)$ & $\mathrm{I}, 438.00(2,005.85)$ & 0.56 \\
(kcal/kg/week) & & & \\
HGS (kg/f) & $17.99(4.2 \mathrm{I})$ & $16.36(3.80)$ & 0.34 \\
GS (m/s) & $0.70(0.16)$ & $0.83(0.18)$ & $0.0 I^{*}$ \\
ALM/h ${ }^{2}$ & $6.53(0.77)$ & $4.97(0.28)$ & $0.0 I^{*}$ \\
\hline
\end{tabular}

Notes: *Significant difference between the groups. Data are expressed as mean ( \pm standard deviation).

Abbreviations: NSE, non-sarcopenic elderly; SE, sarcopenic elderly; BMI, body mass index; HGS, handgrip strength; kg/f, kilogram/force; GS, gait speed; ALM/h², skeletal muscle mass index. and SE 56.3\%), and labyrinth dysfunctions (NSE 33.3\% and SE 28.1\%).

The muscular performance of knee extensors between the groups was statistically different with regard to power and total work, normalized by body weight at a rate of $180^{\circ} \mathrm{s}$ ( $P=0.01$ and $P=0.01$, respectively) (Figures 2 and 3 ). Further analysis of muscle performance showed no statistically significant difference between groups $(P>0.05$; Table 2; Figure 4). Regarding the analysis of plasma levels of inflammatory mediators, there was a statistically significant difference between the groups with regard to the concentration of sTNFR1 $(P=0.01)$, but no difference was observed for IL-6 $(P=0.39)$ (Figure 5).

\section{Discussion}

This study aimed to compare the measures of muscular performance and plasma levels of IL- 6 and sTNFR1 between 

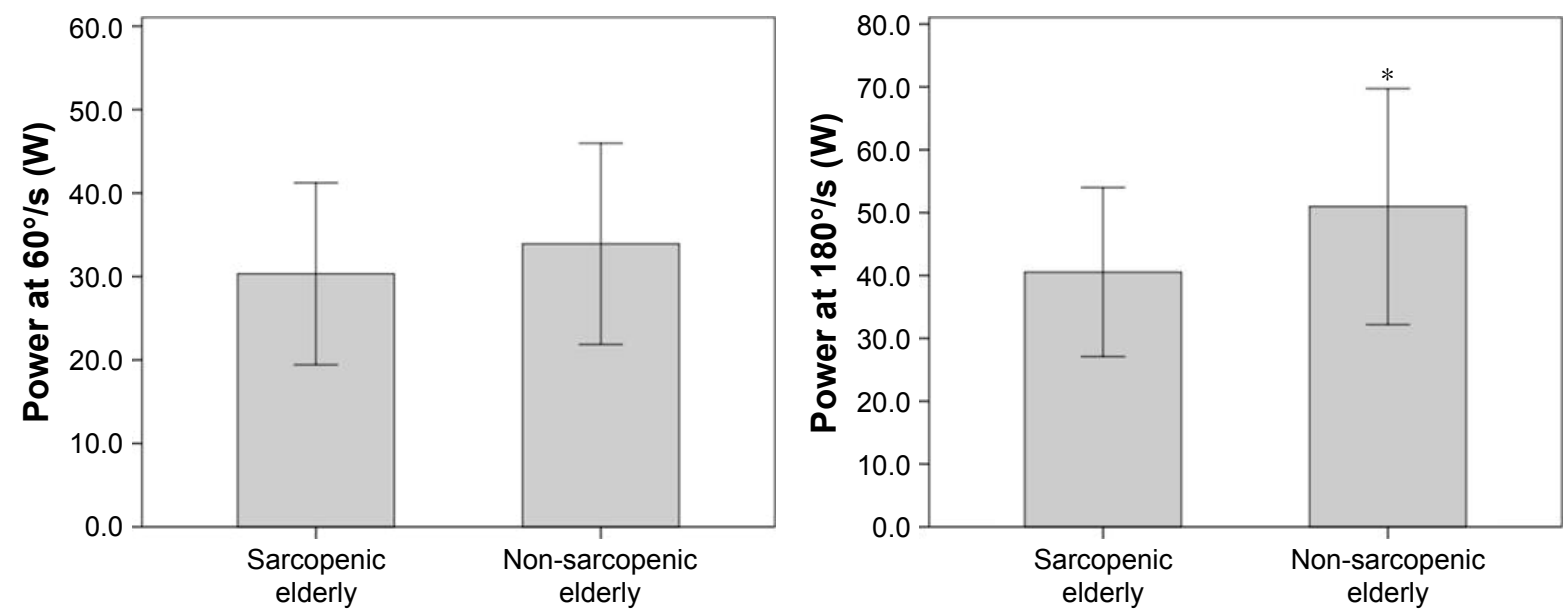

Figure 2 Performance of sarcopenic elderlies and non-sarcopenic elderlies with regard to power at $60 \%$ s (W) and I80\% (W)

Note: *Significant differences between the groups.

SE and NSE women. The results showed homogeneity between groups, except for BMI and number of comorbidities (higher in the NSE group). The SE group showed lower performance with regard to power and total work normalized by body weight at a rate of $180^{\circ} / \mathrm{s}$ and with regard to plasma levels of sTNFR1 (higher in the NSE group).

When analyzing the outcome of sarcopenia, it is necessary to first understand the relationship between the diagnostic criteria involved in this syndrome. It is known that reduced muscle mass is one of the factors that contribute to decreased muscular function and the relationship between these parameters is nonlinear. In terms of clinical relevance, muscular function is associated with functional disability and other adverse health outcomes in elderly patients, since the isolated loss of muscle mass has a weak or no association with these outcomes. ${ }^{3,21,22}$ The literature suggests that objective measures of muscle performance provided by the isokinetic dynamometer, such as torque peak and power, are strong predictors of mobility restriction in community-dwelling elderlies. In addition, power is associated with functional activities in elderlies and its loss occurs at an earlier age. ${ }^{17}$ This study showed significantly lower values of power and total work at a speed of $180^{\circ} / \mathrm{s}$ in the SE group compared to the NSE group. This difference might be due to a greater loss of type II muscle fiber, which is responsible for higher speed contraction, and it might have an impact on functional activities. ${ }^{3,6}$ The absence of statistical difference of the torque peak between the groups, at both speeds, may be because of this measure was expressed as the force output at a single point of the movement, which does not reflect the muscle activity as a whole, especially when considering the presence of sarcopenia.

Furthermore, although the isokinetic dynamometer is considered the gold standard for assessing muscle
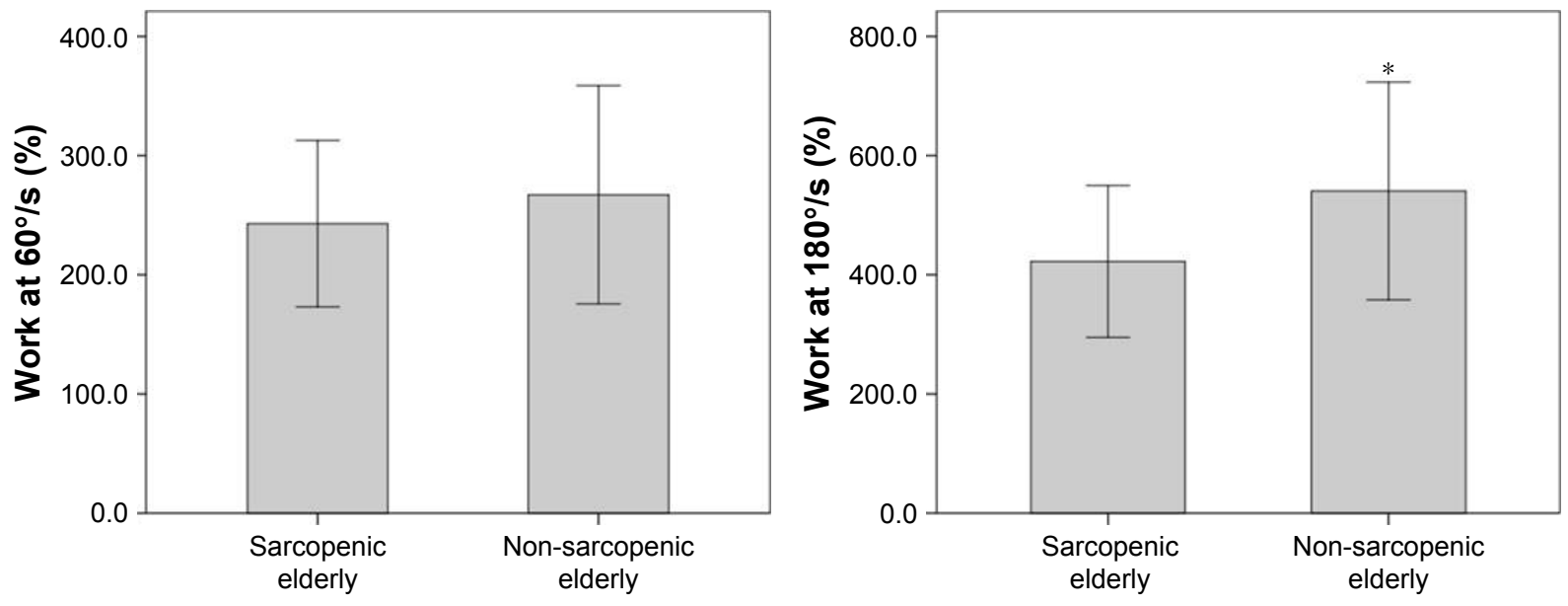

Figure 3 Performance of sarcopenic elderlies and non-sarcopenic elderlies with regard to work normalized by body weight at $60 \% \mathrm{~s}(\%)$ and $180 \% \mathrm{~s}$ (\%). Note: *Significant differences between the groups. 
Table 2 Mean values and standard deviation or average of the analyzed variables and comparison of muscular performance (right limb) and inflammatory mediators between SE and NSE groups

\begin{tabular}{|c|c|c|c|}
\hline Variables & NSE $(n=32)$ & SE $(n=31)$ & $P$-value \\
\hline \multicolumn{4}{|l|}{ Inflammatory mediators } \\
\hline IL-6 (pg/mL), average & 1.92 & 1.54 & 0.39 \\
\hline sTNFRI (pg/mL), average & $2,249.00$ & $1,844.50$ & $0.01 *$ \\
\hline \multicolumn{4}{|c|}{ Muscle performance } \\
\hline Power at $60 \% \mathrm{~s}(\mathrm{~W})$ & $33.92(12.06)$ & $30.33(10.90)$ & 0.22 \\
\hline Power at $180^{\circ} / \mathrm{s}(\mathrm{W})$ & $50.96(18.77)$ & $40.53(13.43)$ & $0.01 *$ \\
\hline $\begin{array}{l}\text { Work normalized by body } \\
\text { weight at } 60 \% \text { s (\%) }\end{array}$ & $267.05(91.52)$ & $242.84(69.77)$ & 0.24 \\
\hline $\begin{array}{l}\text { Work normalized by body } \\
\text { weight at } 180 \% \text { s (\%) }\end{array}$ & $540.49(182.67)$ & $422.36(127.34)$ & $0.01 *$ \\
\hline Torque peak at $60 \% \mathrm{~s}(\mathrm{~J})$ & $54.40(17.27)$ & $49.22(15.47)$ & 0.09 \\
\hline Torque peak at $180 \%$ s $(\mathrm{J})$ & $31.06(8.82)$ & $26.40(6.87)$ & 0.09 \\
\hline
\end{tabular}

Notes: *Significant differences between the groups; muscle performance measures are expressed as mean ( \pm standard deviation).

Abbreviations: NSE, non-sarcopenic elderly; SE, sarcopenic elderly; IL-6, interleukin-6; sTNFRI, soluble receptors of tumor necrosis factor alpha.

performance, ${ }^{3}$ the evaluation of these different parameters (torque peak, total work, and power) of sarcopenia outcome is scarce. To our knowledge, only one study evaluated the relationship of muscle performance of the lower limbs (torque peak parameter of knee extensors) by means of an isokinetic dynamometer at various angular speeds $\left(0^{\circ}, 60^{\circ}\right.$, $120^{\circ}$, and $180^{\circ} / \mathrm{s}$ ) in elderlies with sarcopenia. ${ }^{23}$ The authors found higher torque peak values of knee extensors in NSE group at all speeds, ${ }^{23}$ which was not confirmed in the present study. Moreover, differences in higher speeds $\left(180^{\circ} / \mathrm{s}\right)$, particularly with regard to power, shown in this study may be associated with the ability to perform functional tasks, which meets the diagnostic criteria of EWGSOP, reinforcing the importance to investigate other muscle performance parameters apart from torque peak in the elderlies. ${ }^{2,3}$

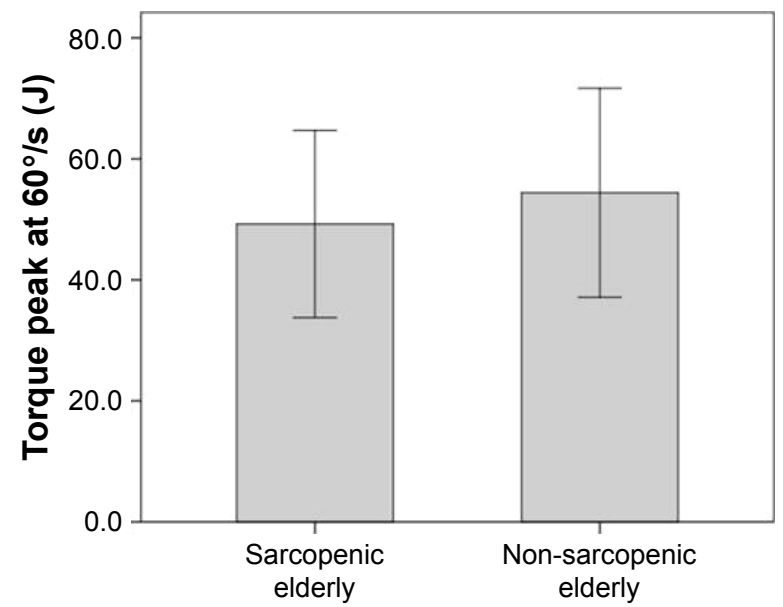

Several studies have reported that increased plasma concentration of IL-6 and sTNFR1 is inversely proportional to the muscle mass parameters, muscle strength, and physical function. ${ }^{7}$ However, based on our knowledge, this is the first study to investigate the relationship between inflammatory mediators and outcome of sarcopenia, based on the algorithm proposed by EWGSOP. In the present study, there was a significant difference in the levels of sTNFR1 between the groups, with lower values in the SE group (NSE $2,249 \mathrm{pg} / \mathrm{mL}$ and SE $1,844.5 \mathrm{pg} / \mathrm{mL}, P=0.01)$. In normal conditions of immune defense, sTNFR1 exerts inhibitory function and assists in the regulation of TNF-alpha activity, and its production is also mediated by this cytokine itself, among others. ${ }^{78}$ In situations such as infections, sTNFR1 reflects TNF-alpha activity, but in cases such as sepsis, of higher clinical magnitude, the concentration of sTNFR1 seems to reach a plateau, not being able to follow the higher plasma levels of TNF-alpha. ${ }^{9}$

The chronic inflammatory increasing process characteristic of aging, is associated with the high concentration of cytokine dosages, IL-6 and TNF-alpha, and their receptors, as sTNFR1, even in the absence of chronic diseases. However, in chronic conditions, TNF-alpha is associated with muscle catabolism (eg, cancer, heart disease, and chronic obstructive pulmonary disease), but the existing mechanisms are not yet clearly understood. ${ }^{7,9}$ In such case, one might think that in sarcopenia, as a chronic condition, sTNFR1 could be associated with high concentrations of TNF-alpha, with insufficient ability to track their activity throughout time. However, this is just a speculation at the moment and it should be confirmed by future studies.

Studies reported in the literature suggest that sTNFR1 may be associated with the modulation of the inflammatory

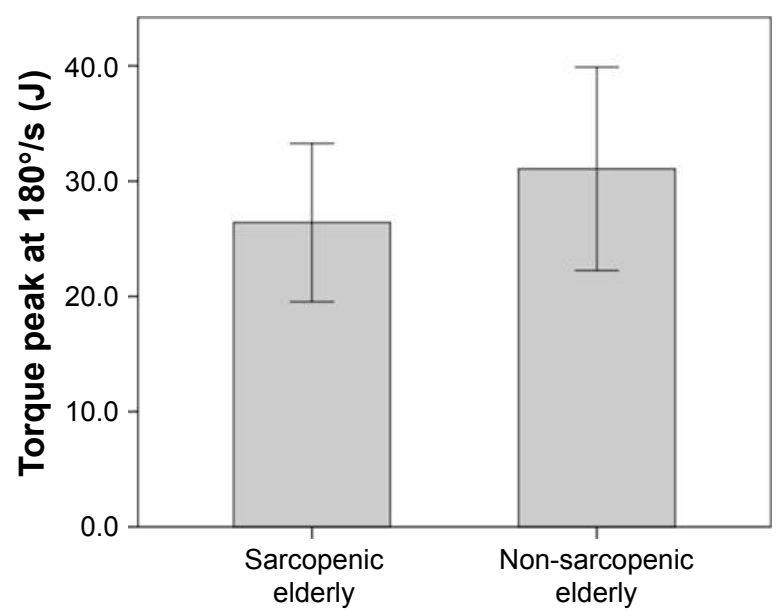

Figure 4 Performance of sarcopenic elderlies and non-sarcopenic elderlies with regard to torque peak at $60 \% / \mathrm{s}(\mathrm{J})$ and $180 \% \mathrm{~s}(\mathrm{~J})$. 

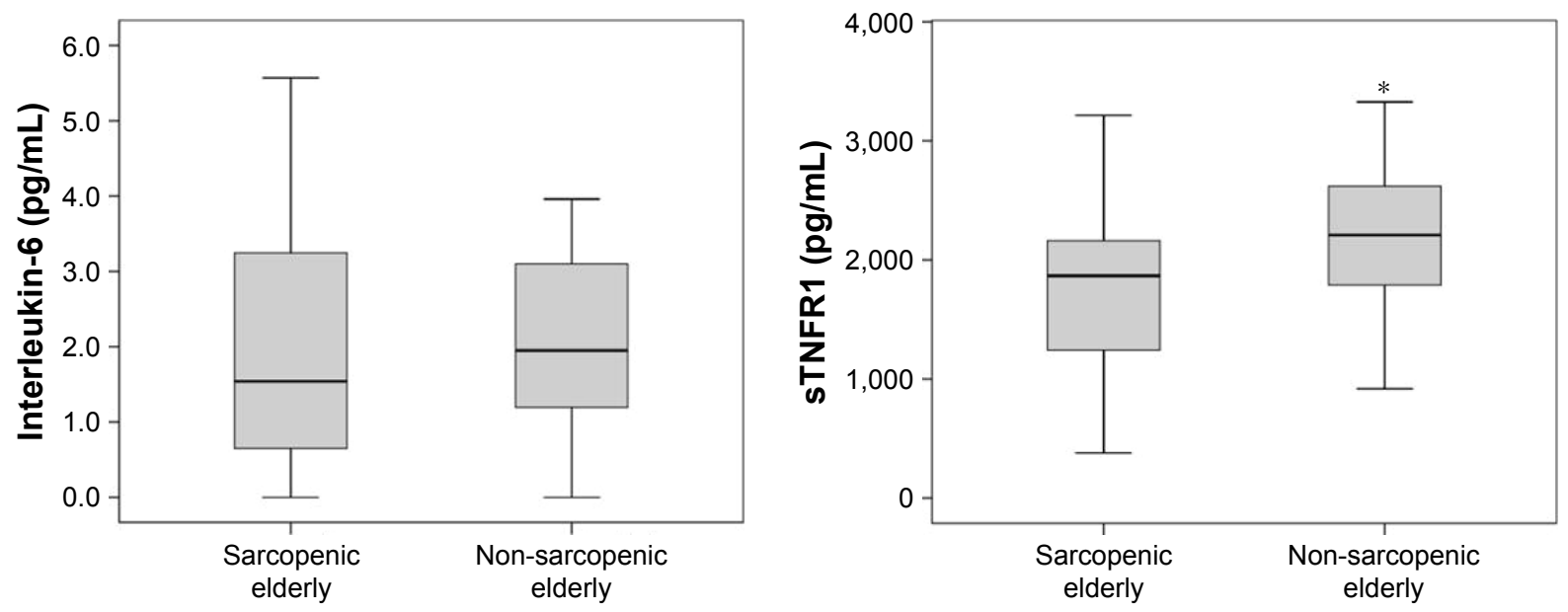

Figure 5 Values of interleukin-6 (pg/mL) and soluble receptors of tumor necrosis factor alpha (sTNFRI; pg/mL) of sarcopenic elderlies and non-sarcopenic elderlies. Note: *Significant differences between the groups.

process, and in specific situations, such as physical exercise, it may be related to the reduction of pro-inflammatory profile in elderlies. ${ }^{7,9,21,24}$ The fact that the present study did not investigate TNF-alpha concentrations prevents the verification of the relationship between the modulatory role of sTNFR1 in inflammatory process and sarcopenia outcome.

Schaap et al reported a significant association between high levels of sTNFR1 in the plasma and loss of muscle mass and strength, evaluated by computed tomography and Jamar dynamometer, in a follow-up of 5 years. ${ }^{11}$ Gonzalo-Calvo et al found a significant association of IL-6 and sTNFR1 with low functional capacity, and this finding was independent of confounding factors such as age, gender, anti-inflammatory drugs, and comorbidities. ${ }^{25}$ Similarly, other studies found a significant association of high concentrations of IL-6 and plasma sTNFR1 with muscle function. ${ }^{26-29}$ However, as these authors did not investigate differences between groups and did not include the integration between the parameters, muscle mass, muscular function and/or functional performance, it is not possible to compare these results to diagnose sarcopenia. However, it appears that high concentrations of sTNFR1 can influence muscle performance, ${ }^{24,25}$ which should be further investigated in the future.

This study found no significant difference between groups regarding IL-6. Barbieri et al reported a combination of high doses of IL-6 with reduced muscle power and muscular function, suggesting that IL-6 concentrations $\geq 1.73 \mathrm{pg} / \mathrm{mL}$ should be considered as a risk factor that reduces muscle function and power, thereby causing disabilities in elderlies. ${ }^{27}$ A dosage of $2.5 \mathrm{pg} / \mathrm{mL}$ of IL- 6 predicts functional disability in community-dwelling elderlies without previous functional restriction. ${ }^{27,28}$ Considering the reference values associated with deleterious effects on the functionality, Ferrucci et al suggested values of $3.1 \mathrm{pg} / \mathrm{mL}$ for IL-6 plasma concentrations in community-dwelling elderly with moderate to severe functional disability to follow the cohort for 3 years. ${ }^{28,29}$

Although IL-6 is a cytokine widely studied in the field of gerontology and is associated with several adverse outcomes in the elderlies, no adverse events were observed in this particular population. One factor often associated with high IL-6 levels is the highest concentration of adipose tissue. ${ }^{7}$ In this study, the NSE group had higher BMI index compared to SE (NSE 28.48 \pm 5.31 and SE 21.55 $\pm 2.28, P=0.01$ ), which might be due to higher concentration of IL- 6 and sTNFR1. Thus, adipose tissue is considered a dynamic endocrine organ, linked to the chronic inflammatory condition in the elderlies. ${ }^{7,29}$ It produces abundant inflammatory mediators associated with inflammation (including IL-6 and sTNFR1) and is positively associated with body weight, BMI, and waist and hip circumferences. ${ }^{29}$ It is worth remembering the possibility of sarcopenic obesity, common in the elderlies, which is related to a clinical picture with interrelatedness of a higher percentage of fat, weight loss, and muscle function. ${ }^{30}$ In such case, one might think that high levels of IL-6, TNF-alpha, and sTNFR1 could be the precursors of this condition.

This assumption is further reinforced by the observation of a higher number of self-reported chronic diseases in the NSE group compared to SE (NSE 2.82 \pm 1.59 and SE 1.94 $\pm 1.21, P=0.02$ ), and the presence of these comorbidities per se already contributes to the inflammatory processes related to aging, thereby influencing the plasma levels investigated in this study.

This study has some limitations. Sample size was calculated based on the diagnostic variables of sarcopenia. In 
the current sample, the operating criterion reduction of muscle mass proved to be an important factor for sarcopenia classification according to the algorithm proposed by EWGSOP. The index for muscle loss adopted in this study, widely used in the literature and adjusted by height, may have underestimated sarcopenic elderlies with higher BMI values. ${ }^{21,22}$ However, because inflammatory biomarkers, particularly IL-6, are not a stable measure to be considered, defining the sample size based on this variable may have contributed to type II error. In this way, caution should be taken to interpret the findings related to inflammatory mediators. There was no significant differences between groups for BMI and number of comorbidities. Nevertheless, the higher BMI values and larger numbers of comorbidities in NSE group may have influenced the behavior of plasma concentrations of IL-6 and sTNFR1. We should also consider that the cutoff points of diagnostic parameters of sarcopenia are related to distinct populations of Brazil, and it is known that socioclinical and environmental differences of the elderlies can influence the behavior of the diagnostic criteria and the clinical course of sarcopenia. In addition, due to the methodological design of the study, it is not possible to infer the causality among the variables studied. Moreover, it is worth considering in future studies the possibility of analyzing elderly individuals who may be in the stages of pre-sarcopenia, sarcopenia, and severe sarcopenia, as suggested by the EWGSOP. .,3 $^{2,}$

\section{Conclusion}

This study showed that sarcopenic elderly presented worse knee extensor performance, especially at a velocity rate of $180 \%$ s. These results suggest that sarcopenia actually impairs muscle performance, thereby affecting the functional activities of daily living, especially in tasks that involve greater agility, and thus predisposes to a greater vulnerability to postural instability and falls. The absence of statistical difference with regard to IL-6 inflammatory marker between NSE and SE and the presence of lower plasma levels of sTNFR1 in the SE might have been influenced by the higher BMI and greater number of comorbidities in the NSE group. Future studies should focus on understanding the role of inflammatory mediators in sarcopenia.

\section{Acknowledgments}

We are grateful to Dr Adriana Maria Kakehasi and staff, for their valuable advice and assistance with DXA exams. This study was sponsored by Pró-Reitoria de Pesquisa da Universidade Federal de Minas Gerais, Belo Horizonte, MG, Brazil, and Conselho Nacional de Desenvolvimento
Científico e Tecnológico (CNPq), Fundação de Amparo à Pesquisa do Estado de Minas Gerais (FAPEMIG).

\section{Disclosure}

The authors report no conflicts of interest in this work.

\section{References}

1. Zhong S, Chen CN, Thompson LV. Sarcopenia of ageing: functional, structural and biochemical alterations. Rev Bras Fisioter. 2007;11(2): 91-97.

2. Cruz-Jentoft AJ, Landi F, Topinkova E, Michel JP. Understanding sarcopenia as a geriatric syndrome. Curr Opin Clin Nutr Metab Care. 2010;13(1):1-7.

3. Cruz-Jentoft AJ, Baeyens JP, Bauer JM, et al; European Working Group on Sarcopenia in Older People. Sarcopenia: European consensus on definition and diagnosis: report of the European Working Group on Sarcopenia in Older People. Age Ageing. 2010;39(4):412-423.

4. Diz JBM, Leopoldino AAO, Moreira BS, et al. Prevalence of sarcopenia in older Brazilians: a systematic review and meta-analysis. Geriatr Gerontol Int. 2017;17(1):5-16.

5. Cruz-Jenftoft AJ, Landi F, Schneider SM, et al. Prevalence of and interventions for sarcopenia in ageing adults: a systematic review. Report of the International Sarcopenia Initiative (EWGSOP and IWGS). Age Ageing. 2014;43(6):748-759.

6. Doherty TJ. Invited review: aging and sarcopenia. J Appl Physiol. 2003; 95(4):1717-1727.

7. Singh $\mathrm{T}$, Newman AB. Inflammatory markers in population studies of aging. Ageing Res Rev. 2011;10(3):319-329.

8. von Haehling S, Steinbeck L, Doehner W, Springer J, Anker SD. Muscle wasting in heart failure: an overview. Int J Biochem Cell Biol. 2013;45(10):2257-2265.

9. Brockhaus M. Soluble TNF receptor: what is the significance? Intensive Care Med. 1997;23(8):808-809.

10. Coelho FM, Narciso FMS, Oliveira DM, et al. sTNFR-1 is an early inflammatory marker in community versus institutionalized elderly women. Inflamm Res. 2010;59(2):129-134.

11. Schaap LA, Pluijm SMF, Deeg DJH, et al. Higher inflammatory marker levels in older persons: associations with 5-year chance in muscle mass and muscle strength. J Gerontol A Biol Sci Med Sci. 2009; 64(11):1183-1189.

12. Brinkley TE, Leng X, Miller ME, et al. Chronic inflammation is associated with low physical function in older adults across multiple comorbidities. J Gerontol A Biol Sci Med Sci. 2009;64A(4):455-461.

13. Legrand D, Adriaensen W, Vaes B, Mathei C, Wallemacq P, Degryse J. The relationship between grip strength and muscle mass (MM) inflammatory biomarkers and physical performance in community-dwelling very old persons. Arch Gerontol Geriatr. 2013;57(3): 345-351.

14. Bertolucci PHF, Brucki SMD, Campacci SR, Juliano Y. O mini-exame do estado mental em uma população geral. [The Mini-Mental State Examination in a general population:impact of educational status]. Arq de Neuro-psiquiatr. 1994;52(1):1-7.

15. Lusardi MM. Is walking speed a vital sign? Absolutely. Top Geriatr Rehabil. 2012;28(2):67-76.

16. Bohannon RW. Comfortable and maximum walking speed of adults aged 20-79 years: reference values and determinants. Age Ageing. 1997;26(1):15-19.

17. Roberts HC, Denison HJ, Martin HJ, et al. A review of the measurement of grip strength in clinical and epidemiological studies: towards a standardised approach. Age Ageing. 2011;40(4):423-429.

18. Cawthon PM. Assessment of lean mass and physical performance in sarcopenia. J Clin Densitom. 2015;18(4):467-471.

19. Baumgartner RN, Koehler KM, Gallagher D, Romero L, Heymsfield SB, Ross RR, et al. Epidemiology of sarcopenia among the elderly in New Mexico. Am J Epidemiol. 1998;147(8):755-763. 
20. Lustosa LP, Pereira LS, Coelho FM, et al. Impact of an exercise program on muscular and functional performance and plasma levels of interleukin 6 and soluble receptor tumor necrosis factor in prefrail community-dwelling older women: a randomized controlled trial. Arch Phys Med Rehabil. 2013;94(4):660-666.

21. Haack M, Pollmacher T, Mullington JM. Diurnal and sleep-wake dependent variations of soluble TNF- and IL-2 receptors in healthy volunteers. Brain Behav Immun. 2004;18(4):361-377.

22. Dam TT, Peters KW, Fragala M, et al. An evidence-based comparison of operational criteria for the presence of sarcopenia. J Gerontol A Biol Sci Med Sci. 2014;69(5):584-590.

23. Marsh AP, Miller ME, Saikin AM, et al. Lower extremity strength and power are associated with 400-meter walk time in older adults: the InCHIANTI study. J Gerontol A Biol Sci Med Sci. 2006;61(11): 1186-1193.

24. Merriwether EM, Host HH, Sinacore DR. Sarcopenic indices in community-dwelling older adults. J Geriatr Phys Ther. 2012;35(3): $118-125$.

25. Gonzalo-Calvo D, Luxán-Delgado B, Rodríguez-González S, et al. Interleukin 6, soluble tumor necrosis factor receptor I and red blood cell distribution width as biological markers of functional dependence in an elderly population: a translational approach. Cytokine. 2012;58(2): 193-198.
26. Hsu FC, Kritchevsky SB, Liu Y, et al. Association between inflammatory components and physical function in the health, aging, and body composition study: a principal component analysis approach. $J$ Gerontol A Biol Sci Med Sci. 2009;64(5):581-589.

27. Barbieri M, Ferrucci L, Ragno E, et al. Chronic inflammation and the effect of IGF-I on muscle strength and Power in older persons. Am J Physiol Endocrinol Metab. 2002;284(3):E481-E487.

28. Ferrucci L, Harris TB, Guralnik JM, et al. Serum IL-6 level and the development of disability in older persons. J Am Geriatr Soc. 1999;47(6): 639-646.

29. Ferrucci L, Pennix BW, Volpato S, et al. Change in muscle strength explains accelerated decline of physical function in older women with high interleukin-6 serum levels. J Am Geriatr Soc. 2002;50(12):1947-1954.

30. Park HS, Park JY, Yu R. Relationship of obesity and visceral adiposity with serum concentrations of CRP, TNF-alpha and IL-6. Diabetes Res Clin Pract. 2005;69(1):29-35.
Clinical Interventions in Aging

\section{Publish your work in this journal}

Clinical Interventions in Aging is an international, peer-reviewed journal focusing on evidence-based reports on the value or lack thereof of treatments intended to prevent or delay the onset of maladaptive correlates of aging in human beings. This journal is indexed on PubMed Central, MedLine,

\section{Dovepress}

CAS, Scopus and the Elsevier Bibliographic databases. The manuscript management system is completely online and includes a very quick and fair peer-review system, which is all easy to use. Visit http://www.dovepress. $\mathrm{com} /$ testimonials.php to read real quotes from published authors. 